\title{
Can Vascular Injury be Appropriately Assessed With Physical Examination After Knee Dislocation?
}

\author{
Douglas S. Weinberg MD, Nicholas R. Scarcella BS, Joshua K. Napora MD, \\ Heather A. Vallier MD
}

Received: 7 October 2015/Accepted: 27 January 2016/Published online: 4 February 2016

(C) The Association of Bone and Joint Surgeons (B) 2016

\begin{abstract}
Background Knee dislocations are rare injuries with potentially devastating vascular complications. An expeditious and accurate diagnosis is necessary, as failing to diagnose vascular injury can result in amputation; however, the best diagnostic approach remains controversial.

Questions/purposes We asked: (1) What patient factors are predictors of vascular injury after knee dislocation? (2) What are the diagnostic utilities of palpable dorsalis pedis or posterior tibial pulses, and the presence of an anklebrachial index $(\mathrm{ABI})$ of 0.9 or greater?
\end{abstract}

Each author certifies that he or she, or a member of his or her immediate family, has no funding or commercial associations (eg, consultancies, stock ownership, equity interest, patent/licensing arrangements, etc) that might pose a conflict of interest in connection with the submitted article.

All ICMJE Conflict of Interest Forms for authors and Clinical Orthopaedics and Related Research ${ }^{\mathbb{R}}$ editors and board members are on file with the publication and can be viewed on request.

Each author certifies that his or her institution approved the human protocol for this investigation, that all investigations were conducted in conformity with ethical principles of research, and that informed consent for participation in the study was obtained.

This investigation was performed at MetroHealth Medical Center, Cleveland, $\mathrm{OH}$, USA.

D. S. Weinberg ( $\varangle)$, J. K. Napora

Department of Orthopaedic Surgery, University Hospitals, Case

Medical Center, Case Western Reserve University, 11100 Euclid

Avenue, Cleveland, OH 44106, USA

e-mail: dsw56@case.edu

N. R. Scarcella

School of Medicine, Case Western Reserve University, Cleveland, OH, USA

H. A. Vallier

Department of Orthopaedic Surgery, MetroHealth Medical

Center, Cleveland, OH, USA
Methods A database at a Level I trauma center was queried for patients with evidence of knee dislocation, demographic information (age at the time of injury, sex, Injury Severity Score, BMI, mechanism of injury), and the presence of open injury were recorded. One-hundred fortyone patients underwent screening at initial presentation, of whom $26(24 \%)$ underwent early vascular exploration based on an abnormal physical examination. One-hundred five $(91 \%)$ of the remaining 115 patients were available at a minimum followup of 6 months (mean, $19 \pm 10$ months). In total, 31 unique patients were excluded, including 10 patients (7\%) who were lost to followup before 6 months. Among the 110 patients who met inclusion criteria, the mean age and SD was $37 \pm 13$ years, and the Injury Severity Score was $15 \pm 9$. There were 71 males $(65 \%)$. Logistic regression was used to determine independent correlates of vascular injury. The vascular examination was reviewed for the presence of a palpable pulse in the dorsalis pedis artery, the presence of a palpable pulse in the posterior tibial artery, and whether the ABI in the dorsalis pedis was 0.9 or greater. Contingency tables were generated to assess the sensitivity, specificity, and accuracy of physical examination maneuvers. The physical examination was collectively regarded as "normal" when both pulses were palpable and the ABI was 0.9 or greater. The initial physical examination as just described was considered the diagnostic test being evaluated in this study; "positive" tests were evaluated by and confirmed at vascular surgery, and 6 months clinical followup without symptoms or progressive signs of vascular injury confirmed the absence of injury in the remainder of the patients. Contingency tables were generated again to assess the sensitivity, specificity, and accuracy of the combined physical examination. 
Results Increased BMI (odds ratio [OR], 1.077; 95\% CI, $1.008-1.155 ; \mathrm{p}=0.033)$ and open injuries (OR, 3.366; 95\% CI, $1.008-11.420 ; p=0.048$ ) were associated with vascular injury. No single physical examination maneuver had a $100 \%$ sensitivity for ruling out vascular injury. A normal physical examination (palpable pulses and $\mathrm{ABI} \geq 0.9$ ) had $100 \%$ sensitivity for ruling out vascular injury.

Conclusions Increased BMI and the presence of open dislocation are associated with a greater risk for vascular injury after knee dislocation. The combination of a palpable dorsalis pedis and posterior tibial pulse combined with an ABI of 0.9 or greater was $100 \%$ sensitive for the detection of vascular injury based on clinical followup at 6 months.

Level of Evidence Level III, diagnostic study.

\section{Introduction}

Knee dislocations are serious injuries caused by violent trauma. Although uncommon, their consequences may be devastating [10]. They often occur as part of orthopaedic polytrauma and may be associated with other disorders and complications; one of the worst of such is arterial injury, which threatens the loss of life and limb [4, 16, 20]. However, limited predictive information is available to suggest which patients are at greater risk for vascular injury [24].

An expeditious and accurate diagnosis is of paramount importance to avoid limb loss in these critically injured individuals, although the appropriate manner in which to do so remains controversial. Historically, trauma providers relied solely on physical examination to diagnose vascular injury in knee dislocation by assessing for the presence of pulses and, in some cases, ankle-brachial index (ABI) [22]. However, advances in technology have offered an expanded array of radiographic tools. This has muddied the clinical algorithms used as a part of various trauma protocols; the exact role of radiography such as CT angiography, arterial duplex, and MR arthrography has been debated, but not settled $[6,12,15]$. In an increasingly restricted-payer healthcare environment, it would seem prudent to examine the diagnostic utility of advanced radiography and provide an evidence-based analysis of CT angiography. Furthermore, given the changing demographics of our society, it would seem especially prudent to reexamine the risk factors and treatment algorithms for knee dislocation, as much of the existing knowledge in the literature is based on clinical data that are more than three decades old [12].

Accordingly, we designed an experiment to answer the following questions: (1) What patient factors are predictors of vascular injury after knee dislocation? (2) What are the diagnostic utilities of palpable dorsalis pedis or posterior tibial pulses, and the presence of an ABI of 0.9 or greater?

\section{Patients and Methods}

After institutional review board approval, a registry of patients who received care at a Level I trauma center between January 1, 2000, and December 31, 2014, was searched. Search criteria were performed according to International Classification of Diseases, 9th Revision (ICD9) codes and Orthopaedic Trauma Association classifications for patients sustaining dislocations of the tibiofemoral joint. One hundred forty-one patients were identified as candidates for study. Exclusion criteria included patients with incomplete medical records (20), those with less than 6 months of clinical followup (10), incorrect ICD-9 classification (nine), or patients who died before having undergone a physical examination (one). In total, 31 unique exclusions were made leaving 110 patients available for primary analysis.

Information regarding age at the time of injury, sex, Injury Severity Score (ISS), BMI, mechanism of injury (high versus low energy), and the presence of an open injury were documented based on the medical record. Injuries resulting from motorcycle or motor vehicle collisions, falls from height, crush injuries, or injuries in pedestrians struck by vehicles were coded as high-energy mechanisms; all other mechanisms were coded as low energy.

The trauma intake history and physical examination was reviewed by two researchers (DSW, NRS) not involved in patient care. The following components of the vascular examination were recorded: the presence or absence of a palpable pulse in the dorsalis pedis artery, the presence or absence of a palpable pulse in the posterior tibial artery, and whether the ABI in the dorsalis pedis was 0.9 or greater. Doppler probes were used as part of ABI measurements [17]. The physical examination was coded as "abnormal" if either of the pulses were absent or if the ABI was less than 0.9. In all cases, serial examinations were performed for at least 48 hours.

Vascular injury was defined as being positive in patients who has vascular exploration after an abnormal examination, and negative for patients whose clinical followup at a minimum of 6 months suggested the absence of symptoms. The diagnostic utilities for predicting vascular injury were calculated for the following independent variables: the presence of a palpable dorsalis pedis pulse, the presence of a posterior tibial pulse, the presence of dopplerable pulses on examination, and the presence of an ABI of 0.9 or greater. The physical examination was collectively regarded as "normal" when both pulses were palpable and there 
was an ABI of 0.9 or greater. The diagnostic utility of a normal physical examination also was calculated.

Arterial injury was considered major if an occlusion existed in the popliteal artery, medial genicular artery, anterior tibial artery, posterior tibial artery, superficial femoral artery, or common femoral artery. Arterial injuries were considered minor if they were confined to the tunica intima or if isolated vasospasm was present [12].

The mean age and SD for the patients at the time of injury was $37 \pm 13$ years. The average ISS was $15 \pm 9$; there were 71 males $(65 \%)$ and 39 females. The mean BMI was $31 \pm 8 \mathrm{~kg} / \mathrm{m}^{2}$. Twenty-six patients had low-energy injuries (24\%), and 84 sustained injuries from high-energy mechanisms. Thirty-eight patients $(35 \%)$ had injuries that were open and 72 had closed injuries. The mean followup was $19 \pm 10$ months.

A physical examination at the time of initial trauma intake that explicitly documented vascular pulses was present in the electronic medical record for all 110 patients. In total, 26 patients (24\%) eventually were shown to have major vascular injury and minor arterial injury was documented in two patients (2\%). Twenty-three patients $(88 \%$ of those with vascular injury) were found to have occlusions of the popliteal artery, two had occlusions of the anterior tibial artery (8\%), and one had an occlusion of the posterior tibial artery (4\%). Patients who sustained major vascular injuries were treated with bypass graft (21 of 26; $81 \%$ ), primary amputation (three of $26 ; 11 \%$ ), angioplasty (one of 26; 4\%), and medication/observation (one of 26; $4 \%)$. When bypass grafting vascular repair did occur, it occurred at a mean of $8 \pm 8$ hours from the time of injury. There were no patients with a vascular injury that was missed on initial trauma intake that presented with late sequelae.

The SPSS software package (Version 22.0; IBM Corporation, Armonk, NY, USA) was used for all data analysis. Binomial logistic regression was used to determine the independent predictors of vascular injury. Significance was set at a $\mathrm{p}$ value of 0.05 or less. Contingency tables were created to assess the diagnostic utility of the measured physical examination maneuvers. Sensitivities, specificities, and accuracies were calculated.

\section{Results}

Increased BMI was associated with a $7.7 \%$ increase in the odds of sustaining a vascular injury (odds ratio [OR], 1.077; 95\% CI, 1.008-1.155; p = 0.033) (Table 1). Patients with open injuries were more likely to sustain vascular injuries compared with patients with closed injuries (OR, 3.366; 95\% CI, 1.008-11.420; p = 0.048). Age, sex, highenergy injury patterns, laterality, and ISS were not associated with vascular injury, with the numbers available (Table 1).

ABI less than 0.9 (Table 2) had the highest positive likelihood ratio $(81 ; 95 \%$ CI, 17-947) for predicting vascular injury. Correspondingly, it also had the highest specificity (99\%) for predicting vascular injury. The presence of a palpable dorsalis pedis pulse had the lowest negative likelihood ratio $(0.03 ; 95 \% \mathrm{CI}, 0.02-0.30)$. It also had the highest sensitivity for ruling out vascular injury (98\%). There were only two patients whose combined physical examination findings were discordant with the presence of a vascular injury, resulting in a false-positive result in both cases (Table 3 ). The combination of physical examination had a sensitivity of $100 \%$, a specificity of $98 \%$, a positive predictive value of $93 \%$, a negative predictive value of $100 \%$, and an overall diagnostic accuracy of $98 \%$. In other words, there were no patients with a diagnosis of vascular injuries within 6 months of a knee dislocation who had a normal physical examination.

The minor vascular injuries in two patients were both isolated tears of the tunica intima. These patients were managed without formal vascular intervention with one receiving aspirin and clopidogril and the other receiving no medication. Followup at 2.5 and 5 years, respectively, revealed no lingering effects suggestive of vascular injury.

\section{Discussion}

Medina et al. [12] recently performed a systematic review of vascular injury after knee dislocations. They queried 23 articles that included 862 patients with knee dislocations and determined that "no outcome-driven conclusions can be drawn regarding the ideal diagnostic modality [of vascular injury]." As they explained, many of the patients included in their meta-analysis had injuries that occurred more than 30 years ago, and when interpreted in the context of the changing demographics of modern society, it would seem valuable to continue reporting the diagnostic utility of various physical examination maneuvers. Although some authors [18, 21, 24] agree that knee dislocations are best diagnosed and managed through a systematic approach, the specifics of such have been heavily debated [12, 13, 24]. Levy et al. [10] reported a shortage of high-quality evidence from which to base diagnosis and treatment decisions, specifically those related to vascular injury. Routine arteriography has been advocated by some [1, 13], although there has been a gradual shift in opinion toward more selective use of angiography $[6,9,16]$. We therefore performed a review of a large trauma database and asked the following questions: (1) What patient factors are predictors of vascular injury after knee dislocation? (2) What are the diagnostic utilities of 
Table 1. Results, binary logistic regression, and physician-independent predictors of vascular injury

\begin{tabular}{lllcc}
\hline Independent variable & Odds ratio & $95 \%$ CI for odds ratio & B (regression coefficient) & Significance \\
\hline Age & 0.970 & $0.924-1.024$ & -0.029 & 0.255 \\
Sex (female) & 1.004 & $0.281-3.499$ & 0.004 & 0.994 \\
BMI & 1.077 & $1.008-1.155$ & 0.076 & 0.033 \\
Energy (high) & 0.658 & $0.160-2.692$ & -0.410 & 0.570 \\
Laterality of injury (right) & 0.492 & $0.164-1.476$ & -0.710 & 0.206 \\
ISS & 0.990 & $0.921-1.099$ & -0.006 & 0.888 \\
Open injury & 3.366 & $1.008-11.420$ & 1.219 & 0.048 \\
\hline
\end{tabular}

ISS = Injury Severity Score; for dichotomous variables, categories in parenthesis were coded as positive.

Table 2. Positive and negative likelihood ratios assessing the diagnostic accuracy of physical examination maneuvers as predictors of vascular injury

\begin{tabular}{|c|c|c|c|c|c|}
\hline Independent variable & $\begin{array}{l}\text { Positive } \\
\text { likelihood ratio }\end{array}$ & $\begin{array}{l}\text { Negative } \\
\text { likelihood ratio }\end{array}$ & Sensitivity & Specificity & Accuracy \\
\hline Dorsalis pedis pulse & 6.73 & 0.03 & $98 \%$ & $86 \%$ & $88 \%$ \\
\hline Posterior tibial pulse & 4.82 & 0.09 & $92 \%$ & $81 \%$ & $83 \%$ \\
\hline Dopplerable pulses after negative pulses on examination & 8.25 & 0.12 & $89 \%$ & $89 \%$ & $89 \%$ \\
\hline $\mathrm{ABI}<0.9$ & 80.77 & 0.04 & $96 \%$ & $99 \%$ & $98 \%$ \\
\hline
\end{tabular}

$\mathrm{ABI}=$ ankle-brachial index.

Table 3. Observed contingency table for patients' physical examinations

\begin{tabular}{lcc}
\hline Independent variable & Vascular injury & No vascular injury \\
\hline Abnormal dorsalis pedis pulse & 25 & 12 \\
Normal dorsalis pedis pulse & 1 & 73 \\
Abnormal posterior tibial pulse & 24 & 16 \\
Normal posterior tibial pulse & 2 & 68 \\
Abnormal dopplerable dorsalis pedis pulse & 23 & 9 \\
Normal dopplerable dorsalis pedis pulse & 3 & 75 \\
ABI $<0.9$ & 25 & 1 \\
ABI $\geq 0.9$ & 1 & 83 \\
Abnormal physical examination & 26 & 2 \\
Normal physical examination & 0 & 82
\end{tabular}

$\mathrm{ABI}=$ ankle-brachial index.

palpable dorsalis pedis or posterior tibial pulses, and the presence of an ABI of 0.9 or greater?

This study has some limitations. The most important was the lack of a true gold standard on all patients. CT angiography has been well-established as a reliable diagnostic tool for vascular injury in knee dislocation, however, it was not used for all patients in this analysis. We did not assess the utility of CT angiography, although it was studied previously [12]. Instead, we used a reference standard of clinical followup at 6 months from the time of injury. Some authors have speculated that vascular injuries should be expected to present within this time [16, 18, 24]. While we are unaware of any case reports of individuals presenting with vascular injuries after the 6-month time, we acknowledge it may have been possible in our study. It also is possible that any patients who were excluded from analysis may have presented elsewhere with complications or vascular injuries; given the relatively small number of patients in our study; even a few individuals might have influenced the major conclusions. However, this limitation is central to the current study, and all other studies on knee dislocations, as many of these injuries go unrecognized if 
they are spontaneously reduced. Designing a comprehensive study without any missing patients is not possible when dealing with these complicated injuries. Similarly, physical examination techniques and results may have differed between examiners. Injuries to the tunica intima were not considered during contingency analysis and may have been incompletely documented; however, tears of the tunica intima rarely produce vascular occlusion [19, 23, 26] and generally are managed nonoperatively [2]. Our study did not differentiate between the direction of knee dislocations, although posterior dislocations have been shown to have higher rates of vascular injury [12]. Twenty patients were excluded in our study owing to incomplete medical records: these individuals had clinical documentation of knee dislocations, however, complete medical and radiographic records were not available for certain patients, some of whom presented when our institution was transitioning to electronic records, and their files were incomplete. Study design necessitated that patients be excluded if they did not have pulses or ABI recorded during the intake examination.

The collective data from this study suggest that individuals with higher BMI and those with open injuries were more likely to sustain vascular injury after knee dislocation. Age, sex, mechanism of injury, laterality, and ISS did not predict vascular injury. These findings are in accordance with those of Georgiadis et al. [4], who retrospectively identified 53 individuals with knee dislocations and determined that patients who were obese who sustained low-energy falls were more likely to sustain major vascular injuries than patients with high-energy traumatic dislocations. They suggested that the traditionally held risk factors for vascular injury (high-energy mechanism, multiple system trauma) may not be applicable for modern practice, especially as BMIs continue to increase. Sillanpää et al. [21] similarly warned increasing body proportions are leading to a changing paradigm in the epidemiology of knee dislocations and associated vascular injuries. Although historical literature suggested that mechanism of injury and the degree of injury severity (ISS) were useful predictors of vascular injury [7, 8, 10, 14, 25], this was not the case in our study or others [3,21]. To our knowledge, our study is one of the only studies that investigates open injury as a risk factor for vascular injury after knee dislocation; however, Wright et al. [27] reported a series of 19 patients with open knee dislocations and found that nine (47\%) had some form of concomitant vascular injury.

Existing treatment algorithms extrapolated from earlier research have relied on the assessment of the dorsalis pedis pulse, the posterior tibial pulse, the presence of dopplerable pulses, and the assessment of the ABI. We found that the presence of a dorsalis pedis pulse was the most sensitive finding for ruling out a vascular injury in the dislocated knee. Having an ABI less than 0.9 was the most specific physical examination maneuver for detecting the presence of vascular injury; however, although both modalities had excellent statistical accuracy [5], neither had $100 \%$ sensitivity or diagnostic accuracy. Considering the grave clinical consequences of a missed vascular injury [1, 12], any physical examination test with less than $100 \%$ sensitivity for ruling out such a crucial diagnosis is of limited utility when used in isolation. However, Hollis and Daley [6] recommended that physical examination findings should be used in conjunction with one another to provide the most complete clinical picture. No patients in our study who sustained vascular injury $(100 \%$ sensitivity, $100 \%$ negative predictive value) had an entirely normal physical examination. There were two patients who had abnormal physical examinations but did not have any form of vascular injury (two false positives, 93\% specificity, 98\% positive predictive value). However, when the primary purpose of a diagnostic test is to exclude a potentially limbor life-threatening condition, a small number of false positives should be expected and, to some degree, required. A similar concept is used by acute care surgeons during the evaluation of appendicitis, where it has been suggested that a significant false positive rate is necessary to ensure a diagnostic sensitivity of $100 \%$ [5]. The absence of pulses alone has been suggested in some studies $[7,8,10,11,14$, $24,25]$ as being sufficient to detect vascular injury, although each of the studies had limited patient sample size and relied on antiquated technology. Moreover, these studies were limited by their selective use of the ABI, which has been shown to be a valuable adjunct to the vascular examination. Mills et al. [13] prospectively assessed $\mathrm{ABI}$ in 38 patients with knee dislocations and reported $100 \%$ sensitivity and specificity for determining arterial injury that was deemed to be significant when using a cutoff less than 0.9. We agree with the conclusions of Stannard et al. [24], that a full concert of physical examination findings can be used to rule out the presence of vascular injury after knee dislocations. However, a physical examination cannot be used to diagnose the occurrence of a vascular injury, and we agree with Rihn et al. [18] who suggested that angiography is needed to definitively rule in the existence of vascular injury.

Our study corroborates reports suggesting that the conventionally held risk factors for vascular injury after knee dislocation are changing in accordance with the increased size of modern individuals. Increased BMI and the presence of open dislocation are associated with a greater risk for vascular injury after knee dislocation. We found no single physical examination maneuver that was sufficiently sensitive on its own to diagnose vascular injury. However, the combination of a palpable dorsalis pedis and posterior 
tibial pulse combined with an ABI of 0.9 or greater was $100 \%$ sensitive for ruling out vascular injury at a minimum 6-month clinical followup. We recommend that trauma providers evaluate vascular injury after knee dislocation as follows: (1) palpate the dorsalis pedis and posterior tibial artery; (2) perform an $\mathrm{ABI}$; and (3) if both pulses are present and the $\mathrm{ABI}$ is 0.9 or greater, we recommend continued vascular observation for 48 hours [10]. However, if either the dorsalis pedis or posterior tibial pulses cannot be palpated, or if the ABI is less than 0.9, we recommend the use of CT angiography. Similarly, if physical examination findings are at all inconclusive, angiography may be warranted.

Acknowledgments We thank Stephen Bowen BS (University of Virginia, School of Medicine, Charlottesville, VA, USA) for help with data collection.

\section{References}

1. Bui KL, Ilaslan H, Parker RD, Sundaram M. Knee dislocations: a magnetic resonance imaging study correlated with clinical and operative findings. Skeletal Radiol. 2008;37:653-661.

2. Dalman R. Operative Techniques in Vascular Surgery. Philadelphia, PA: Wolters Kluwer Health; 2015.

3. Georgiadis AG, Guthrie ST, Shepard AD. Beware of ultra-lowvelocity knee dislocation. Orthopedics. 2014;37:656-658.

4. Georgiadis AG, Mohammad FH, Mizerik KT, Nypaver TJ, Shepard AD. Changing presentation of knee dislocation and vascular injury from high-energy trauma to low-energy falls in the morbidly obese. J Vasc Surg. 2013;57:1196-1203.

5. Hays WL. Statistics for the Social Sciences. New York, NY: Holt, Rinehart and Winston; 1973.

6. Hollis JD, Daley BJ. 10-year review of knee dislocations: is arteriography always necessary? J Trauma. 2005;59:672-675; discussion 675-676.

7. Kaufman SL, Martin LG. Arterial injuries associated with complete dislocation of the knee. Radiology. 1992;184:153-155.

8. Kendall RW, Taylor DC, Salvian AJ, O'Brien PJ. The role of arteriography in assessing vascular injuries associated with dislocations of the knee. $J$ Trauma. 1993;35:875-878.

9. Klineberg EO, Crites BM, Flinn WR, Archibald JD, Moorman CT 3rd. The role of arteriography in assessing popliteal artery injury in knee dislocations. J Trauma. 2004;56:786-790.

10. Levy BA, Fanelli GC, Whelan DB, Stannard JP, MacDonald PA, Boyd JL, Marx RG, Stuart MJ; Knee Dislocation Study Group. Controversies in the treatment of knee dislocations and multiligament reconstruction. J Am Acad Orthop Surg. 2009;17:197206.
11. Martinez D, Sweatman K, Thompson EC. Popliteal artery injury associated with knee dislocations. Am Surg. 2001;67:165-167.

12. Medina O, Arom GA, Yeranosian MG, Petrigliano FA, McAllister DR. Vascular and nerve injury after knee dislocation: a systematic review. Clin Orthop Relat Res. 2014;472:2621-2629.

13. Mills WJ, Barei DP, McNair P. The value of the ankle-brachial index for diagnosing arterial injury after knee dislocation: a prospective study. J Trauma. 2004;56:1261-1265.

14. Miranda FE, Dennis JW, Veldenz HC, Dovgan PS, Frykberg ER. Confirmation of the safety and accuracy of physical examination in the evaluation of knee dislocation for injury of the popliteal artery: a prospective study. J Trauma. 2002;52:247-251; discussion 251-242.

15. Natsuhara KM, Yeranosian MG, Cohen JR, Wang JC, McAllister DR, Petrigliano FA. What is the frequency of vascular injury after knee dislocation? Clin Orthop Relat Res. 2014;472:26152620.

16. Nicandri GT, Chamberlain AM, Wahl CJ. Practical management of knee dislocations: a selective angiography protocol to detect limb-threatening vascular injuries. Clin $J$ Sport Med. 2009;19:125-129.

17. Ouriel K. Peripheral arterial disease. Lancet. 2001;358:12571264.

18. Rihn JA, Cha PS, Groff YJ, Harner CD. The acutely dislocated knee: evaluation and management. J Am Acad Orthop Surg. 2004;12:334-346.

19. Sawchuk AP, Eldrup-Jorgensen J, Tober C, McCoy D, Durham J, Waller B, Schuler JJ, Flanigan DP. The natural history of intimal flaps in a canine model. Arch Surg. 1990;125:1614-1616.

20. Sharma S, Kumar V, Dhillon MS. A new technique for closed reduction of traumatic posterior dislocations of the hip: the 'PGI technique'. Hip Int. 2014;24:394-398.

21. Sillanpää PJ, Kannus P, Niemi ST, Rolf C, Felländer-Tsai L, Mattila VM. Incidence of knee dislocation and concomitant vascular injury requiring surgery: a nationwide study. J Trauma Acute Care Surg. 2014;76:715-719.

22. Sisto DJ, Warren RF. Complete knee dislocation: a follow-up study of operative treatment. Clin Orthop Relat Res. 1985;198:94-101.

23. Stain SC, Yellin AE, Weaver FA, Pentecost MJ. Selective management of nonocclusive arterial injuries. Arch Surg. 1989;124:1136-1140; discussion 1140-1141.

24. Stannard JP, Sheils TM, Lopez-Ben RR, McGwin G Jr, Robinson JT, Volgas DA. Vascular injuries in knee dislocations: the role of physical examination in determining the need for arteriography. $J$ Bone Joint Surg Am. 2004;86:910-915.

25. Treiman GS, Yellin AE, Weaver FA, Wang S, Ghalambor N, Barlow W, Snyder B, Pentecost MJ. Examination of the patient with a knee dislocation: the case for selective arteriography. Arch Surg. 1992;127:1056-1062; discussion 1062-1063.

26. Welling RE, Kakkasseril J, Cranley JJ. Complete dislocations of the knee with popliteal vascular injury. J Trauma. 1981;21:450453.

27. Wright DG, Covey D, Born CT, Sadasivan KK. Open dislocation of the knee. J Orthop Trauma. 1995;9:135-140. 